\title{
A TAXONOMIA DO ANTIGO GÊNERO ACACIA MILL., COM BASE NA ANÁLISE DE AGRUPAMENTO DE CARACTERES DO LENHO ${ }^{1}$
}

\author{
PAULO FERNANDO DOS SANTOS MACHADO ${ }^{2}$ \\ JOSÉ NEWTON CARDOSO MARCHIORI ${ }^{3}$ ANELISE MARTA SIEGLOCH ${ }^{4}$
}

\begin{abstract}
RESUMO
A análise de agrupamento realizada com caracteres do lenho de 25 espécies do antigo gênero Acacia (Fabaceae: Mimosoideae) permitiu a separação das mesmas nos atuais gêneros Acacia Mill., Senegalia Raf. e Vachellia Wight \& Arn., em concordância com a segregação aprovada pelo Congresso Internacional de Botânica em 2005.

Palavras-chave: Acacia Mill., anatomia da madeira, Senegalia Raf., Vachellia Wight \& Arn., taxonomia da madeira.
\end{abstract}

\section{ABSTRACT}

[The taxonomy of the old genus Acacia Mill., based on cluster analysis of wood characters]. Cluster analysis performed with wood characters of 25 species of the old genus Acacia (Fabaceae: Mimosoideae) allows the separation of the same in the current genera Acacia Mill., Senegalia Raf. and Vachellia Wight \& Arn., in accordance with the segregation approved by the International Botanical Congress in 2005.

Key words: Acacia Mill., Senegalia Raf., Vachellia Wight \& Arn., wood anatomy, wood taxonomy.

\section{INTRODUÇÃO}

A utilização de caracteres da estrutura interna das plantas na classificação vegetal é muito antiga e tem sido utilizada com freqüência (Record, 1944). A esse respeito, Dadswell (1939) pondera que a anatomia da madeira pode contribuir, positivamente, na classificação de gêneros ou famílias de posição taxonômica duvidosa, no estudo do arranjo interno de famílias e na determinação das prováveis afinidades entre famílias botânicas.

1 Recebido em 19-6-2015 e aceito para publicação em 29-8-2015.

2 Engenheiro Florestal, mestrando do Programa de PósGraduação em Engenharia Florestal da Universidade Federal de Santa Maria. Bolsista CNPq. Santa Maria, RS, Brasil.barcasole@gmail.com

3 Engenheiro Florestal, Dr. Bolsista de Produtividade em Pesquisa (CNPq - Brasil). Professor Titular do Departamento de Ciências Florestais, Universidade Federal de Santa Maria. Santa Maria, RS, Brasil.

4 Engenheira Florestal, doutoranda do Programa de PósGraduação em Engenharia Florestal, Universidade Federal de Santa Maria. Bolsista CAPES. Santa Maria, RS, Brasil.
Em anatomia taxonômica, é fundamental distinguir os caracteres anatômicos relativamente constantes, dos passíveis de variação sob diferentes condições de crescimento. A esse respeito, Rendle (1944) pondera ser necessária considerável experiência para reconhecer os caracteres de valor diagnóstico em uma madeira e estimar seu valor como típico de família, gênero, de um táxon infragenérico ou espécie.

A presença de ornamentações em pontoações intervasculares, por exemplo, ocorre em famílias inteiras ou determinados grupos de uma família. Panshin \& De Zeeuw (1970) reportam esse caráter para todas as espécies norteamericanas da família Leguminosae (Fabaceae), com exceção de Cercis L. (Fabaceae: Caesalpinioideae). O número, diâmetro e distribuição das ornamentações também podem variar consideravelmente e ter valor diagnóstico (Bailey, 1951; Ohtani, 1981; Ohtani et al., 1983; Vliet, 1978; Vliet \& Baas, 1984). Por outro lado, caracteres como abundância de poros, comprimento de elementos vasculares e fração de 
parênquima axial são muito influenciados pelo ambiente (Metcalfe \& Chalk, 1972).

No tocante aos raios, o caráter de maior utilidade é a largura, tanto em dimensão linear como em número de células; a altura também pode ser valiosa, principalmente se os mesmos forem ou muito altos ou muito baixos. A presença de raios exclusivamente unisseriados também se revela importante, podendo auxiliar na distinção de gêneros e espécies, sobretudo se o caráter tiver ocorrência esporádica no grupo em questão (Metcalfe \& Chalk, 1972).

O comprimento de elementos vasculares, porosidade (difusa ou em anel), vasos em cadeias ou solitários, presença (ou ausência) de canais intercelulares, tilos, tipos de parênquima axial e estratificação podem ter, igualmente, valor diagnóstico (Titmuss, 1948).

Nas últimas décadas, técnicas estereológicas, associadas à manipulação computadorizada de dados estatísticos, proporcionaram notável contribuição à identificação de madeiras e segregação de espécies afins, além de auxiliar na predição de propriedades físicomecânicas, comportamento tecnológico e uso potencial de madeiras tropicais (Marchiori, 1990). É nesse sentido que a análise de agrupamento hierárquica aglomerativa tem contribuído - e de modo significativo - para a taxonomia botânica, com as vantagens de seguir critério independente, avesso a subjetividades.

O presente artigo, ao realizar uma análise de agrupamento com caracteres anatômicos do lenho, visa, justamente, a investigar, com independência, a taxonomia do antigo gênero Acacia Mill., com base em dados de 25 espécies, recolhidos da literatura.

\section{REVISÃO DE LITERATURA}

Dada a abrangência do presente estudo, que envolve tanto a anatomia do lenho como a taxonomia do gênero Acacia, antes de sua segregação, convém revisar a literatura de ambas as vertentes separadamente, iniciando-se com a taxonomia.
O gênero Acacia foi estabelecido por Philip Miller em 1754, com base em nome proposto por Tournefort (1694). O étimo deriva do grego akakia, um substantivo que se traduz por ponta ou fio (Miller, 1969), e alude à presença conspícua de espinhos no caule e ramos de muitas de suas espécies.

$\mathrm{O}$ antigo gênero achava-se bem representado nas regiões tropicais e subtropicais da América, África, Ásia e Austrália, faltando apenas na Europa e Nova Zelândia (Burkart, 1952). De um total de aproximadamente 1200 espécies, mais da metade (cerca de 700) era procedente do continente australiano (Pedley, 1986).

Na ausência de restos paleontológicos significativos, estudos de morfologia comparada apontam as regiões tropicais do oeste gondwânico, em área do atual continente americano, como centro da diversidade do gênero (Guinet \& Vassal, 1978).

Valendo-se de caracteres do fruto, Willdenow (1805) reafirmou a segregação de Acacia Mill. do gênero Mimosa L., reconhecendo, ainda, os gêneros Desmanthus Willd., Inga Mill. e Schrankia Willd. Para Acacia, o autor relacionou 102 espécies, separadas em seis grupos, de acordo com a morfologia de folhas, inflorescências e presença de acúleos no caule. Com o tempo, muitas espécies por ele citadas passaram a gêneros distintos, entre os quais Albizia Durazz. e Piptadenia Benth.

De Candolle (1825) organizou o gênero Acacia Mill. em quatro seções, com base na morfologia de folhas e inflorescências: Phyllodineae, Conjugatopinnatae, Spiciflorae e Globiflorae.

Fundamentado na ocorrência excludente de filódios e folhas bipinadas, Endlicher (1836) separou Acacia Mill. de Vachellia Wight \& Arn., reconhecendo, para o primeiro, os táxones infragenéricos Rhacospermae e Acacia verae.

Com base em caracteres do androceu, George Bentham - o mais importante leguminólogo do século dezenove - criou a tribo Acacieae em 1842, juntamente com outras cinco da subordem Mimoseae, táxon de extensão correspondente à 
atual subfamília Mimosoideae. Único em sua tribo, o gênero Acacia foi por ele organizado em seis séries fundamentais, com base em caracteres taxonômicos clássicos da morfologia floral e aparelho vegetativo: Phyllodineae, Botryocephalae, Pulchelae, Gummiferae, Vulgares e Filicinae.

Caracterizada pela redução das folhas a filódios, as Phyllodineae compreendiam, originalmente, 204 espécies da Austrália e ilhas do oceano Pacífico (Bentham, 1842). Em revisão posterior, Bentham (1875) ampliou o grupo para 227 espécies, distribuídas em oito subséries.

Igualmente australásica, a série Botryocephalae foi criada para abranger espécies inermes de folhas bipinadas, com capítulos racemosos e pedúnculos solitários. Composta, inicialmente, de treze espécies (Bentham, 1842), o grupo foi reduzido a dez, na revisão de 1875.

Restrita à Austrália e arredores, a série Pulchellae Benth. foi criada com onze espécies de arbustos inermes ou armados de espinhos axilares, providos de folhas bipinadas e flores em capítulos globosos ou espigas cilíndricas, dispostas na axila de folhas ou em racemos terminais pedunculados. Na revisão de 1875 , Bentham conservou apenas oito binômios no grupo.

Composta, inicialmente, por 48 espécies americanas, da África e Ásia, a série Gummiferae foi criada para abranger espécies com folhas bipinadas e estípulas espinescentes, mas desprovidas de acúleos (Bentham, 1845). Na revisão de 1875, o grupo chegou a 60 espécies, agrupadas em três subséries.

Em sua criação, a série Vulgares reunia 53 binômios, distribuídos em cinco subséries (Bentham, 1842). Na revisão de 1875 , o número aumentou para 75 binômios, organizados em quatro subséries, incluindo árvores, arbustos e lianas de folhas bipinadas, com pecíolo glandulífero, estípulas não espinescentes e acúleos infraestipulares esparsos ou ausentes.

A sexta série do antigo gênero Acacia Mill., denominada Filicinae, compunha-se, originalmente, de onze espécies americanas de arbustos inermes, com folhas bipinadas e pecíolo eglanduloso (Bentham, 1842). Na revisão de 1875, o taxonomista inglês conservou apenas duas espécies da "região mexicana". Por seus numerosos caracteres indiferenciados, Guinet \& Vassal (1978) referem-se ao grupo como o mais arcaico de todo o gênero.

De longa vigência, a classificação de George Bentham preponderou na literatura taxonômica por mais de um século, até a reorganização proposta por Vassal (1963, 1972a, b), com base em caracteres filéticos da morfologia da semente e plântulas. O autor restaurou o gênero monotípico Faidherbia (F. albida (Delile) A. Chev.) e segregou as demais espécies em três subgêneros: Aculeiferum Vassal, Acacia Mill. e Heterophyllum Vassal. O primeiro destes (Aculeiferum Vassal), baseia-se nas séries Vulgares e Filicinae, de Bentham. O subgênero Acacia compreende as espécies da série Gummiferae Benth., com exceção de Acacia albida Delile, transferida para o gênero monotípico Faidherbia, como visto anteriormente. Heterophyllum Vassal, por sua vez, reúne as espécies australásicas das séries Phyllodineae, Botryocephalae e Pulchellae, de George Bentham.

Pedley (1986) restaurou o gênero Racosperma (DC.) Mart., transferindo para o mesmo as espécies do subgênero Heterophyllum Vassal, o que implicou na renomeação de centenas de espécies, entre as quais todas as de origem australiana cultivadas no sul do Brasil: Racosperma dealbatum (Link) Pedley (antiga Acacia dealbata Link); Racosperma decurrens (Willd.) Pedley (= Acacia decurrens Willd.); Racosperma mearnsii (De Wild.) Pedley (= Acacia mearnsii De Wild.); Racosperma melanoxylon (R. Br.) Pedley (= Acacia melanoxylon R. Br.); Racosperma podalyriifolium (G. Don.) Pedley (= Acacia podalyriifolia G. Don.). Em artigo posterior, Pedley (2003) apresentou uma sinopse do gênero Racosperma, a qual, entretanto, teve curta vigência, posto que estudos morfológicos e moleculares demonstraram que o antigo gênero Acacia, por ser polifilético, requeria divisão mais radical (Maslin et al., 2003). 
Em 30 de julho de 2005, o Congresso Internacional de Botânica (Viena) endossou a proposta de Orchard \& Maslin (2003), reservando o nome Acacia para o grupo mais numeroso as Phyllodineae, sensu George Bentham -, levando à necessidade de retipificação. Desse modo, a espécie-tipo passou da africana Acacia nilotica (L.) Delile, vinculada à série Gummiferae Benth. (ou subgênero Acacia Mill.), para a australiana Acacia penninervis Sieber ex DC. (McNeil et al., 2005).

No caso das espécies americanas, elas foram transferidas para os gêneros Vachellia Wight \& Arn., Senegalia Raf., Acaciella Britton \& Rose e Mariosousa Seigler \& Ebinger (Seigler \& Ebinger, 2005).

O gênero Acacia Mill. compreende, atualmente, 948 espécies australianas, além de outras dez da Ásia tropical, sete de ilhas do oceano Pacífico e uma ou duas de Madagascar. Das espécies australianas, apenas sete foram transferidas para Vachellia Wight \& Arn. e duas para Senegalia Raf. (Maslin et al., 2003).

O gênero Vachellia Wight \& Arn. reúne todas as espécies do subgênero Acacia Vassal (ou série Gummiferae, sensu George Bentham), posto ser um grupo monofilético. De distribuição pantropical, compreende, atualmente, 163 espécies, das quais 159 são americanas e apenas duas australianas.

O gênero Senegalia Raf. foi segregado do subgênero Aculeiferum Vassal (= série Vulgares Bentham), após comprovação de sua origem polifilética. Com distribuição pantropical, reúne 203 espécies, atualmente, 53 das quais são nativas do continente americano (Rico-Arce, 2007) e apenas duas da Austrália.

O gênero Acaciella Britton \& Rose abrange quinze espécies americanas vinculadas à antiga série Filicinae Benth.

O gênero Mariosousa Seigler \& Ebinger, por sua vez, foi segregado da série Aculeiferum Benth. para abrigar treze espécies americanas afins a Acacia coulteri Benth. (atual Mariosousa coulteri (Benth.) Seigler \& Ebinger).
Com relação à anatomia do lenho, cabe salientar, de início, que a literatura ainda é relativamente escassa, face à importância econômica e extensão do antigo gênero Acacia Mill. Record \& Hess (1949), por exemplo, não fazem qualquer referência ao mesmo em sua obra mais conhecida (Timbers of the New World), apesar das numerosas espécies nativas nas três Américas.

Em estudo de treze espécies argentinas, Cozzo (1951) refere-se ao gênero Acacia como "estruturalmente homogêneo", por não haver encontrado diferenças anatômicas importantes no grupo. No mesmo trabalho, constam detalhes estruturais das treze espécies investigadas e, inclusive, uma chave dicotômica para a identificação das mesmas.

Em pesquisa com espécies sul-africanas, Robbertse et al. (1980) também observaram escassa variação anatômica entre as mesmas, salientando a dimensão dos raios e, com menor importância, a formação de anéis de crescimento distintos, número de poros $/ \mathrm{mm}^{2}$ e distribuição do parênquima axial, como os detalhes mais importantes para a distinção de subgêneros: todas as 14 espécies investigadas do subgênero Aculeiferum Vassal tinham raios estreitos (2-5seriados), em contraste com as 23 espécies do subgênero Acacia Mill., separadas do grupo anterior por terem raios mais largos (4-14seriados).

Ao contrário dos trabalhos anteriormente comentados, Marchiori (1990) observou diferenças "bastante expressivas", em estudo descritivo de quinze espécies nativas e cultivadas no Rio Grande do Sul, chegando a comparar esta diversidade anatômica com a "registrada entre espécies de diferentes gêneros e tribos de Mimosoideae". Apesar do universo restrito investigado pelo autor, foram reconhecidos três grupos com base na anatomia do lenho, coincidentes com os subgêneros de Vassal (1972b).

Com base em 14 espécies nativas e cultivadas no estado do Rio Grande do Sul, Marchiori $\&$ Santos (2011) reconheceram diferenças es- 
truturais suficientes para a identificação anatômica dos atuais gêneros Acacia, Senegalia e Vachellia, corroborando a segregação dos mesmos do antigo gênero Acacia Mill., aprovada no Congresso Internacional de Botânica realizado em Viena, no ano de 2005. Comprovouse que as cinco espécies investigadas do subgênero Aculeiferum Vassal, correspondente ao atual gênero Senegalia Raf., separaram-se das demais pela simples presença de fibras septadas. Por sua vez, as seis espécies do subgênero Heterophyllum Vassal (atual gênero Acacia Mill.) distinguiram-se das três espécies examinadas do gênero Vachellia Wight \& Arn. (subgênero Acacia, sensu Vassal) pelo parênquima axial relativamente escasso $(<20 \%$ do lenho), disposto em séries de 2-4 células. As três espécies investigadas de Vachellia Wight \& Arn., em contraste, apresentaram parênquima axial abundante (> 25\%) e contrastado no plano transversal em relação ao tecido fibroso, compondo séries de duas (raramente três) células de altura.

\section{MATERIAL E MÉTODOS}

O material em análise consiste de dados quantitativos e qualitativos de 25 espécies do antigo gênero Acacia Mill., colhidos de artigos anteriormente publicados por um ou mais autores da presente pesquisa. A relação dos nomes científicos atualmente válidos e dos respectivos binômios reduzidos à sinonímia é apresentada na Tabela 1, juntamente com o subgênero ao qual estavam inseridas as distintas espécies antes da segregação aprovada em 2005, pelo Congresso Internacional de Botânica (Viena, Áustria).

As espécies investigadas e os respectivos artigos em que foram publicados são listados, na sequência, segundo ordem alfabética dos binômios válidos: Acacia acuminata (Machado et al., 2015a); Acacia cambagei (Machado et al., 2015a); Acacia dealbata (Marchiori, 2011b); Acacia decurrens (Marchiori, 2011a); Acacia harpophylla (Machado et al., 2015a); Acacia koa (Machado et al., 2015a); Acacia longifolia (Illana \& Marchiori, 1980); Acacia mearnsii (Costa \& Marchiori, 1980); Acacia melanoxylon (Marchiori, 2009); Acacia podalyriifolia (Marchiori, 2010); Acacia rhodoxylon (Machado et al., 2015a); Acacia richii (Machado et al., 2015a); Senegalia bonariensis (Marchiori, 1996); Senegalia martiusiana (Machado et al., 2015b); Senegalia nitidifolia (Marchiori, 1990, 1991); Senegalia recurva (Marchiori, 1982); Senegalia tucumanensis (Marchiori 1994); Senegalia velutina (Marchiori, 1990, 1995); Senegalia visco (Pereira et al., 2015); (Vachellia caven (Marchiori, 1992); Vachellia collinsii (Machado et al., 2015c); Vachellia farnesiana (Marchiori, 2010b); Vachellia ibirocayensis (Marchiori, 1990, 1993); Vachellia nilotica (Machado et al., 2015c); e Vachellia planifrons (Machado et al., 2015c).

Para comparar e reunir as espécies investigadas em grupos de afinidade, realizouse uma análise de agrupamento hierárquica aglomerativa com base em caracteres anatômicos reconhecidos como de valor taxonômico para o antigo gênero Acacia Mill. (Tabela 2), os quais, com exceção do tamanho de cristais, foram anteriormente utilizados por Marchiori \& Santos (2011), em investigação anatômico-taxonômica preliminar.

Esta análise foi gerada a partir de uma matriz de distância de dissimilaridade de Jaccard, sendo utilizado para a geração dos grupos o Método de Peso Proporcional (UPGMA). Também se calculou o coeficiente de correlação cofenético $(r)$ para validar o dendrograma resultante.

Os dados foram processados no software $\mathrm{R}$ (R Core Team, 2012), pacote VEGAN (Oksanen et al., 2012). Para a validação do dendrograma resultante, calculou-se o coeficiente de correlação cofenético ( $r$ de Pearson). A interpretação e discussão dos resultados levaram em consideração a distribuição das espécies e gêneros no dendrograma, em relação ao arranjo tradicional e as mais recentes classificações internas do grupo (Tabela 1). 
TABELA 1 - Espécies investigadas e respectivos subgêneros botânicos.

Nome científico

Subgênero

(sensu Vassal)

Acacia acuminata A. Gray

[Acacia acuminata A. Gray]

Heterophyllum

Acacia cambagei R.T. Baker

[Acacia cambagei R.T. Baker]

Heterophyllum

Acacia dealbata Link

[Acacia dealbata Link]

Heterophyllum

Acacia decurrens Willd.

[Acacia decurrens Willd.]

Heterophyllum

Acacia harpophylla F. Muell. ex Benth.

[Acacia harpophylla F. Muell. ex Benth.] Heterophyllum

Acacia koa A. Gray

[Acacia koa A. Gray]

Heterophyllum

Acacia longifolia (Andrews) Willd.

[Acacia longifolia (Andrews) Willd.]

Heterophyllum

Acacia mearnsii De Wild.

[Acacia mearnsii De Wild.]

Heterophyllum

Acacia melanoxylon $\mathrm{R}$. Br.

[Acacia melanoxylon R. Br.]

Heterophyllum

Acacia podalyriifolia A. Cunn.

[Acacia podalyriifolia A. Cunn.]

Heterophyllum

Acacia rhodoxylon Maiden

[Acacia rhodoxylon Maiden]

Heterophyllum

Acacia richii A. Gray

[Acacia richii A. Gray]

Heterophyllum 
Senegalia bonariensis (Gillies) Seigler \& Ebinger

[Acacia bonariensis (Gillies ex Hook. \& Arn.]

Aculeiferum

Senegalia martiusiana (Steud.) Seigler \& Ebinger

[Acacia martiusiana (Steud.) Burk.]

Aculeiferum

Senegalia nitidifolia (Speg.) Seigler \& Ebinger

[Acacia nitidifolia Speg.]

Aculeiferum

Senegalia recurva (Benth.) Seigler \& Ebinger

[Acacia recurva Benth.]

Aculeiferum

Senegalia tucumanensis (Griseb.) Seigler \& Ebinger

[Acacia tucumanensis Griseb.]

Aculeiferum

Senegalia velutina (DC.) Seigler \& Ebinger

[Acacia velutina $\mathrm{DC}$.]

Aculeiferum

Senegalia visco (Lorentz ex Griseb.) Seigler \& Ebinger

[Acacia visco Lorentz ex Griseb.]

Aculeiferum

Vachellia caven (Molina) Seigler \& Ebinger

[Acacia caven (Molina) Molina]

Acacia

Vachellia collinsii (Saff.) Seigler \& Ebinger

[Acacia collinsii Saff.]

Acacia

Vachellia farnesiana (L.) Wight \& Arn.

[Acacia farnesiana (L.) Willd.]

Acacia

Vachellia ibirocayensis (Marchiori) Deble \& Marchiori

[Acacia ibirocayensis Marchiori].

Acacia

Vachellia nilotica (L.) P.J.H. Hurter

[Acacia nilotica L.]

Acacia

Vachellia planifrons (Wight \& Arn.) Ragup, Seigler,

Ebinger \& Maslin

[Acacia planifrons Wight \& Arn.]

Acacia

Os binômios entre colchetes, constantes em obras mais antigas, caíram em sinonímia.

Observar que as espécies australianas não sofreram alteração, neste sentido. 
TABELA 2 - Distribuição das características anatômicas nas espécies em estudo.

\begin{tabular}{|c|c|c|c|c|c|c|c|c|}
\hline \multirow[b]{2}{*}{ Espécies } & \multicolumn{8}{|c|}{ Caracteres } \\
\hline & PAe & PAa & SP1 & SP2 & $\mathrm{Re}$ & $\mathrm{Rl}$ & Fs & Fns \\
\hline A. acuminata Benth. & 1 & 0 & 0 & 1 & 1 & 0 & 0 & 1 \\
\hline A. cambagei R. T. Baker & 1 & 0 & 1 & 0 & 1 & 0 & 0 & 1 \\
\hline A. dealbata Link & 1 & 0 & 0 & 1 & 1 & 0 & 0 & 1 \\
\hline A. decurrens Willd. & 1 & 0 & 0 & 1 & 1 & 0 & 0 & 1 \\
\hline A. harpophylla F. Muell. Ex Benth & 1 & 0 & 1 & 0 & 1 & 0 & 0 & 1 \\
\hline A. koa A. Gray & 1 & 0 & 1 & 0 & 1 & 0 & 0 & 1 \\
\hline A. longifolia Paxton & 1 & 0 & 0 & 1 & 1 & 0 & 0 & 1 \\
\hline A. mearnsii De Wild. & 1 & 0 & 0 & 1 & 1 & 0 & 0 & 1 \\
\hline A. melanoxylon $\mathrm{R} . \mathrm{Br}$. & 1 & 0 & 0 & 1 & 1 & 0 & 0 & 1 \\
\hline A. podalyriifolia A. Cunn. & 1 & 0 & 0 & 1 & 1 & 0 & 0 & 1 \\
\hline A. rhodoxylon Maiden & 1 & 0 & 0 & 1 & 1 & 0 & 0 & 1 \\
\hline A. richii A. Gray & 1 & 0 & 0 & 1 & 1 & 0 & 0 & 1 \\
\hline S. bonariensis (Gillies) Seigler \& Ebinger & 1 & 0 & 0 & 1 & 1 & 0 & 1 & 0 \\
\hline S. martiusiana (Stend.) Seigler \& Ebinger & 1 & 0 & 1 & 0 & 1 & 0 & 1 & 0 \\
\hline S. nitidifolia (Spreg.) Seigler \& Ebinger & 1 & 0 & 0 & 1 & 1 & 0 & 1 & 0 \\
\hline S. recurva (Benth.) Seigler \& Ebinger & 1 & 0 & 0 & 1 & 1 & 0 & 1 & 0 \\
\hline S. tucumanensis (Griseb.) Seigler \& Ebinger* & 1 & 0 & 1 & 0 & 1 & 0 & 1 & 0 \\
\hline S. velutina (DC.) Seigler \& Ebinger* & 1 & 0 & 0 & 1 & 1 & 0 & 1 & 0 \\
\hline S. visco (Lorentz ex Griseb.) Seigler \& Ebinger & 1 & 0 & 0 & 1 & 1 & 0 & 1 & 0 \\
\hline V. caven (Molina) Seigler \& Ebinger & 0 & 1 & 0 & 1 & 0 & 1 & 0 & 1 \\
\hline V. collinsii (Saff.) Seigler \& Ebinger & 1 & 0 & 1 & 0 & 0 & 1 & 0 & 1 \\
\hline V. farnesiana Wight \& Arn. & 0 & 1 & 0 & 1 & 0 & 1 & 0 & 1 \\
\hline V. ibirocayensis (Marchiori) Deble \& Marchiori & 0 & 1 & 0 & 1 & 1 & 0 & 0 & 1 \\
\hline V. nilotica (L.) P.J.H. Hunter \& Mabb. & 1 & 0 & 0 & 1 & 0 & 1 & 0 & 1 \\
\hline V. planifrons (Wight \& Arn.) Ragup., Seigler, Ebinger \& Maslin & 0 & 1 & 0 & 1 & 0 & 1 & 0 & 1 \\
\hline
\end{tabular}

Onde: $\mathrm{PAe}=$ parênquima axial escasso $(<20 \%) ; \mathrm{PAa}=$ parênquima axial abundante $(>25 \%) ; \mathrm{SP} 1$ = parênquima axial com séries de 2-4-8 células; SP2 = parênquima axial com séries de 2-4 células; $R$ e = raios estreitos (14 células de largura); $\mathrm{Rl}=$ raios largos ou conspícuos $(+6$ células de largura); Fs = fibras septadas; Fns = fibras não septadas; *arbusto; Caráter presente $=1$; Caráter ausente $=0$.

\section{RESULTADOS E DISCUSSÃO}

Até 2005, todas as espécies do presente estudo incluíam-se no antigo gênero Acacia Mill. Sob o ponto de vista anatômico, observa-se em todo o conjunto um grande número de caracteres anatômicos em comum, salientando-se: elementos vasculares curtos a médios; placas de perfuração simples; pontoações intervasculares alternas; pontoações ornamentadas pequenas a mé- dias; parênquima paratraqueal; raios homogêneos; células procumbentes; fibras libriformes curtas a médias; e presença de cristais. Mais do que peculiares ao antigo gênero Acacia Mill., essas características têm ampla ocorrência na subfamília Mimosoideae, repetindo-se em numerosos gêneros.

Apesar da referida homogeneidade estrutural, o Dendrograma da Figura 1 permite separar as espécies investigadas em três grupos distin- 
tos, em concordância com a atual concepção taxonômica. Para a construção do mesmo, utilizaram-se caracteres anatômicos destacados por Marchiori (1990), Marchiori \& Santos (2011) e Robbertse et al. (1980) como importantes para a segregação de grupos de espécies em Acacia Mill.

De acordo com a Figura 1, vê-se que o grupo de espécies de Senegalia liga-se ao das atuais Acacia, e que essa proximidade anatômica se deve ao compartilhamento de raios estreitos e parênquima axial escasso. Ambos os grupos, por sua vez, separam-se, facilmente, com base na presença ou ausência de fibras septadas, respectivamente.

Mais diversificado, o grupo formado pelas espécies de Vachelia apresenta maior heterogeneidade, comparado aos dois anteriores. O parênquima axial é abundante em Vachellia caven, V. farnesiana, V. ibirocayensis e $V$. planifrons, mas escasso em V. collinsii e $V$. nilotica. Por sua vez, Vachellia caven, $V$. farnesiana, V. nilotica e V. planifrons apresen- tam raios largos, distintamente de $V$. ibirocayensis, que tem raios estreitos. Importa salientar, por fim, que não se observaram fibras septadas em nenhuma espécie do gênero Vachellia e que as mesmas apresentam parênquima axial em séries de duas, menos comumente quatro células de altura.

Ao reconhecer, com base na estrutura do lenho, a existência de três grupos de espécies no antigo gênero Acacia Mill., o presente estudo contesta a homogeneidade estrutural atribuída por Cozzo (1951), corrobora resultados anteriores de Marchiori (1980, 1990), Marchiori \& Santos (2011) e Robbertse et al. (1980), e vêm reforçar a segregação taxonômica do mesmo, aprovada pelo Congresso Internacional de Botânica no ano de 2005. Os gêneros Acacia Mill., Senegalia Raf. e Vachellia Wight \& Arn., em outras palavras, distinguem-se, facilmente, entre si - e não apenas por detalhes da morfologia externa -, como, também, por caracteres anatômicos do lenho.

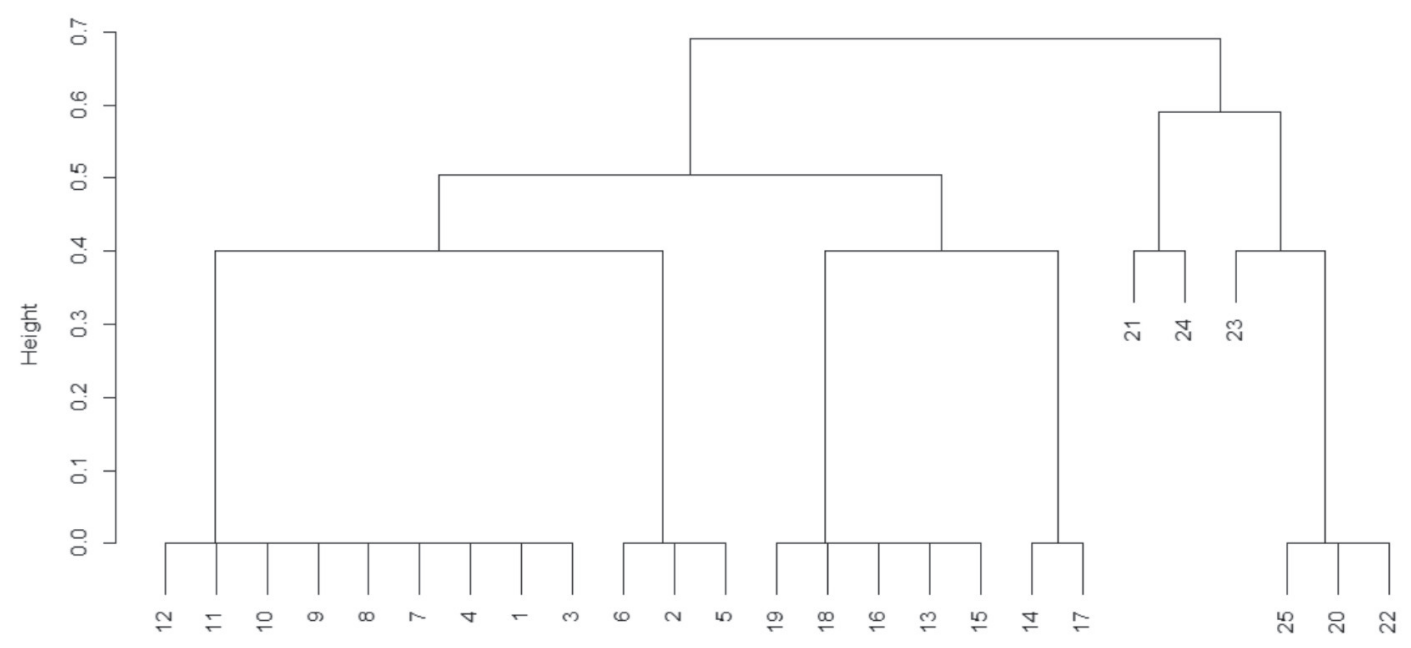

FIGURA 1 - Dendrograma (Jaccard, UPGMA) das 25 espécies investigadas. 1 - A. acuminata; 2 - A. cambagei; 3 - A. dealbata; 4 - A. decurrens; 5 - A. harpophylla $; 6-A$. koa; 7 - A. longifolia; 8 - A. mearnsii; 9 - A. melanoxylon; $10-$ A. podalyriifolia; $11-A$. rhodoxylon; $12-A$. richii; $13-$ S. bonariensis; $14-S$. martiusiana; $15-S$. nitidifolia $; 16-S$. recurva; 17 - S. tucumanensis*; 18 - S. velutina*; 19 - S. visco; 20 - V. caven; 21 - V. collinsii; 22 - V. farnesiana; 23 - V. ibirocayensis; 24 - V. nilotica; 25 - V. planifrons. $r=0,88$; *arbusto. 


\section{REFERÊNCIAS BIBLIOGRÁFICAS}

BAILEY, I.W. The use and abuse of anatomical data in the study of phylogeny and classification. Phytomorphology, v. 1, p. 67-69, 1951.

BENTHAM, G. Notes on Mimoseae, with a synopsis of species. The London Journal of Botany, n. 1, p. 318-392, 494-518, 1842.

BENTHAM, G. Revision of the sub-order Mimoseae. Trans. Linn. Soc. London, n. 30, p. 335-664, 1875.

BURKART, A. Las Leguminosas argentinas silvestres y cultivadas. Buenos Aires ACME Agency, 1952. $569 \mathrm{p}$.

COSTA, A.F. da; MARCHIORI, J.N.C. Estudo anatômico da madeira de Acacia mearnsii De Willd. In: Anais do IV Congresso Florestal Estadual, Nova Prata, Rio Grande do Sul, 1980. p. 237-245.

COZZO, D. Anatomia del leño secundario de las Leguminosas Mimosoideas y Cesalpinoideas argentinas silvestres y cultivadas. Rev. Inst. Nac. Invest. Ci. Nat. Ci. Bot., Buenos Aires, v. 2, n. 2 , p. 63-290, 1951.

DADSWELL, H.E. The role of wood anatomy in forest botany. J. Counc. Sci. Indust. Res. Aust., v. 12, p. 237-242, 1939.

DE CANDOLLE, A.P. Prodromus Systematis Naturalis Regni Vegetabilis. Paris: Treuttel \& Würtz, 1825. v. 2. 644p.

ENDLICHER, S.L. Genera Plantarum Secundum Ordines Naturales Disposita. Vienna: F. Beck, 1836-1840. 1483p.

GUINET, P.; VASSAL, J. Hypotheses on differentiation of the major groups in the genus Acacia (Leguminosae). Kew Bulletin, v. 32, p. 509-527, 1978.

ILLANA, H.A.; MARCHIORI, J.N.C. Estudo anatômico do xilema secundário de Acacia longifolia (Andr.) Willd. In: Anais do IV Congresso Florestal Estadual, Nova Prata, Rio Grande do Sul, 1980. p. 207-215.

MACHADO, P.F. dos S.; MARCHIORI, J.N.C.; SIEGLOCH, A.M. Anatomia da madeira de seis espécies exóticas do gênero Acacia Mill. (Fabaceae). Balduinia, Santa Maria, n. 49, p. 1$15,2015 \mathrm{a}$

MACHADO, P.F. dos S.; MARCHIORI, J.N.C.; SIEGLOCH, A.M. Anatomia do lenho de Senegalia martiusiana (Steud.) Seigler \& Ebinger. Balduinia, Santa Maria, n. 49, p. 2428, 2015b.

MACHADO, P.F. dos S.; MARCHIORI, J.N.C.; SIEGLOCH, A.M. Anatomia do lenho de três espécies exóticas do gênero Vachellia Wight \& Arn. Balduinia, Santa Maria, n. 50, p. 1-10, 2015.

MARCHIORI, J.N.C. Estudo anatômico do xilema secundário e da casca de algumas espécies dos gêneros Acacia e Mimosa, nativas no estado do Rio Grande do Sul. Curitiba: UFPR, 1980. 185 f. Dissertação (Mestrado em Engenharia Florestal - Universidade Federal do Paraná).

MARCHIORI, J.N.C. Estudo anatômico do xilema secundário e da casca de Acacia recurva Benth. (Leguminosae Mimosoideae). Ciência e Natura, Santa Maria, n. 4, p. 95-105, 1982.

MARCHIORI, J.N.C. Anatomia das madeiras do gênero Acacia, nativas e cultivadas no estado do Rio Grande do Sul. Curitiba: UFPR, 1990. 226f. Tese (Doutorado em Engenharia Florestal - Universidade Federal do Paraná).

MARCHIORI, J.N.C. Anatomia da madeira de Acacia nitidifolia Speg. (Leguminosae Mimosoideae). Ciência Florestal, Santa Maria, v.1, n. 1, p. 46-63, 1991.

MARCHIORI, J.N.C. Anatomia da madeira e casca do espinilho, Acacia caven (Mol.) Mol. Ciência Florestal, Santa Maria, v. 2, n. 1, p. 27-47, 1992.

MARCHIORI, J.N.C. Estudo anatômico do xilema secundário de Acacia ibirocayensis Marchiori (Leguminosae Mimosoideae). Ciência e Natura, Santa Maria, n. 15, p. 149-159, 1993.

MARCHIORI, J.N.C. Anatomia da madeira e casca de Acacia tucumanensis Gris. Ciência e Natura, Santa Maria, n. 16, p. 85-104, 1994.

MARCHIORI, J.N.C. Anatomia da madeira e casca de Acacia velutina DC. Ciência e Natura, Santa Maria, n. 17, p. 99-114, 1995.

MARCHIORI, J.N.C. Anatomia da madeira de Acacia bonariensis Gill. ex Hook. \& Arn. Ciência Rural, Santa Maria, v. 26, n. 2, p. 209-216, 1996.

MARCHIORI, J.N.C. Anatomia da madeira de Acacia melanoxylon R. Br. Balduinia, Santa Maria, n. 18, p. 26-32, 2009.

MARCHIORI, J.N.C. Anatomia da madeira de Acacia podalyriifolia A. Cunn. Balduinia, Santa Maria, n. 20, p. 10-15, 2010a.

MARCHIORI, J.N.C. Anatomia da madeira de Vachellia farnesiana (L.) Wight \& Arn. Balduinia, Santa Maria, n. 22, p. 9-14, 2010 b.

MARCHIORI, J.N.C. Anatomia da madeira de Acacia decurrens Willd. Balduinia, Santa Maria, n. 26, p. 1-7, 2011a.

MARCHIORI, J.N.C. Anatomia da madeira de Acacia dealbata Link. Balduinia, Santa Maria, n. 29 , p. 21-27, 2011 b. 
MARCHIORI, J.N.C.; SANTOS, S.R. dos. A segregação do gênero Acacia (Tourn.) Mill., sob o ponto de vista da anatomia da madeira de espécies nativas e cultivadas no Rio Grande do Sul. Balduinia, Santa Maria, n. 30, p. 25-36, 2011.

MASLIN, B.R.; MILLER, J.T.; SEIGLER, D.S. Overview of the generic status of Acacia (Leguminosae: Mimosoideae). Australian Systematic Botany, v. 16, p. 1-18, 2003.

MAcNEILL, J.; STUESSY, T.F.; TURLAND, N.J.; MÖRANDL, E. XVII International Botanical Congress: preliminary mail vote and report of Congress action on nomenclature proposals. Taxon, v. 54, n. 4, p. 1057-1064, 2005.

METCALFE, C.R.; CHALK, L. Anatomy of Dicotyledons. Oxford: Clarendon Press, $1972.1500 \mathrm{p}$.

MILLER, P. The gardeners dictionary. New York: J. Cramer, 1969. 1582 p. (Abridged ed. 1754; Historia Naturalis Classica, LXXII).

OHTANI, J. Vesture or warts. Proposed terminology. IAWA Bulletin, v. 5, n. 1, p. 37-40, 1981.

OHTANI, J.; MEYLAN, B.A.; BUTTERFIELD, B.G. Occurrence of warts in the vessel elements and fibres of New Zealand woods. New Zealand Journal of Botany, n. 21, p. 359-372, 1983.

OKSANEN, J.; BLANCHET, F.G.; KINDT, R.; LEGENDRE, P.; O'HARA, R.B.; SIMPSON, G.1.; STEVENS, M.H.H.; WAGNER, H. vegan: community ecology package. Version 2.0-6. 2012 Avaiable from: <http://vegan.r-forge.rproject.org/>.

ORCHARD, A.E.; MASLIN, B.R. Proposal to conserve the name Acacia (Leguminosae: Mimosoideae) with a conserved type. Taxon, v. 52, p. 362-363, 2003.

PANSHIN, A.J.; DE ZEEUW, C. Textbook of wood technology. New York: McGraw-Hill, 1970. v. $1.705 \mathrm{p}$.

PEDLEY, L. Derivation and dispersal of Acacia (Leguminosae), with particular reference to Australia, and the recognition of Senegalia and Racosperma. Bot. J. Linn. Soc., Londres, n. 92, p. 219-254, 1986.

PEDLEY, L. Synopsis of Racosperma. Austrobaileya, v. 6, p. 445-496, 2003.

PEREIRA, A.C. da S.; SIEGLOCH, A.M.; MARCHIORI, J.N.C. Anatomia do lenho de Senegalia visco (Lorentz ex Griseb.) Seigler \& Ebinger (Fabaceae). Balduinia, Santa Maria, n. 47, p. 19-23, 2015.
RECORD, S.J. Role of the wood anatomy in taxonomy. Tropical woods, n. 37, p. 1-9, 1934.

RECORD, S.J.; HESS, R.W. Timbers of the New World. New Haven: Yale University Press, 1949. $640 \mathrm{p}$.

RENDLE, B.J. On the taxonomic value of the anatomical structure of the vegetative organs of Dicotyledons. 3. The role of anatomy in the practical identification of commercial timbers. Proc. Linnean Soc. London, v. 155, n. 3, p. 218221, 1944.

RICO-ARCE, M de L. American species of Acacia. México: Comisión Nacional para el Conocimiento y Uso de la Biodiversidad (CONABIO), 2007. 207 p.

ROBBERTSE, P.J.; VENTER, G.; RENSBURG, H.J. van. The wood anatomy of the south African Acacias. IAWA Bulletin, v. 1, n. 3, p. 93-103, 1980.

SEIGLER, D.S.; EBINGER, J.E. New combination in the genus Vachellia (Fabaceae: Mimosoideae) from the New World. Phytologia, v. 87, n. 3, p. 139-178, 2005 (publ. 2006).

TITMUS, F.H.A. Concise encyclopaedia of world timbers. London: Mechanical Press, 1948. 156 p.

TOURNEFORT, P. Elemens de Botanique ou methode pour conôtre les plantes. Paris: Imprimerie Royale, 1694. 379 p.

VASSAL, J. Interêt taxonomique de la morphologie des graines dans le genre Acacia. Bull. de la Societé d'Histoire Naturelle, Toulouse, v. 98, n. 3-4, p. 341-371, 1963.

VASSAL, J. Ontogenetic and seed research applied to the morphological, taxonomical and phylogenetic study of the genus Acacia. Travaux Lab. For. Toulouse, v. 1, n. 8, 1972a. 125 p.

VASSAL, J. Apport des recherches ontogénetiques et séminologiques à l'étude morphologique, taxonomique et phylogénique du genre Acacia. Bull. Soc. Hist. Nat., Toulouse, v. 108, p. 125247, $1972 b$.

VLIET, G.J.C.M. van. The vestured pits of Combretaceae and allied families. Acta Bot. Neerlandica, n. 27, p. 273-285, 1978.

VLIET, G.J.C.M. van; BAAS, P. Wood anatomy and classification of the Myrtales. Ann. Missouri Bot. Garden, n. 71, p. 783-800, 1984.

WILLDENOW, C.L. Species Plantarum. Paris : Berlin: 1805 p. 1049-1093. (Editio quarta, Tomus IV). 